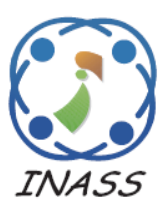

\title{
Extending the Neural Model to Study the Impact of Effective Area of Optical Fiber on Laser Intensity
}

\author{
Kavita Bhatnagar $^{1 *}$ \\ Subhash Chander Gupta ${ }^{2}$ \\ ${ }^{1}$ Jamia Millia Islamia, Delhi, India \\ ${ }^{2}$ Raj Kumar Goel Institute of Technology, Uttar Pradesh, India \\ * Corresponding author's Email: kavitabhatnagar56@gmail.com
}

\begin{abstract}
Our previous article has proposed a simulation model to explore the nonlinear relationship between the nonlinear regime of the fiber, laser intensity and the error probability of the optical link. This paper extends the model to include the effective area of the fiber. In order to handle the extended parameter, time series feed forward neural network based prediction model is proposed. The reliability of the model is substantiated by extensive experimental analysis through which the relationship exhibiting between the laser intensity and the corresponding nonlinear regime and effective area of the fiber can be observed. The observed results demonstrate the performance of the prediction model in estimating the launch power to meet the desired error probability under varying nonlinear regime and effective of the fiber.
\end{abstract}

Keywords: Nonlinear regime, Effective area, Optical link, Time series, Launch power.

\section{Introduction}

Coherent optical fiber systems, which incorporate analog-to-digital converters (ADCs), digital-to-analog converters (DACs) and digital signal processors that operate with extreme speed [1] [2]. Nevertheless, the optical communication link is posed with the problem of optical fiber non-linearity [3]. Moreover, the fiber non-linearity could cause more restrictions on the limits of the fundamental Shannon capacity [4-6]. Compensation of the nonlinear distortion may be probably accomplished, if nonlinear pre-distortion as well as OPC transmitter are utilized [7].

There are plenty of factors, which help in defining the nonlinearity effects that are causes in the optical fibers [8]. Yet, only a small number of researches have been conducted to examine the optical fiber nonlinearity, in association with the factor s. In [8], it has been stated that the radiation effects related with the optical source are of major concern in describing the optical fiber nonlinearity. This implies that the definition of the optical fiber nonlinearity has a massive linkage with the optical source's radiation effects. The high coherent nature of the lasers has caused the magnitude of the optical fiber nonlinearity to be large. The characterization of the fiber nonlinearity depending on the intensity of the laser is more vital, whenever the nonlinearities are supposed to be compensated or decreased. To the best of our knowledge, no research contributions have been reported in the literature to characterize the fiber nonlinearities using systematic analysis and mathematical models.

This paper performs an extensive analysis on the fiber properties that leads for fiber nonlinearity. The analysis is extended from our previous work in which the launch power has been determined based on the nonlinear regime of the fiber to accomplish the minimum bit error rate for the system. Here, we study the impact of the effective area of the fiber along with the nonlinear regime. The impact of an effective area of fiber and its nonlinearity also vary with the optical power intensity in the fiber. Besides, the nonlinear regime specifically pulses that can efficiently and selectively stimulate two-photon transitions in order to attain an arbitrarily phase, 
amplitude, and polarization-shaped pulses after propagation through a step-index fiber. Hence, the proposed neural model can effectively represent the characteristics of the fiber link, which is not reported in the literature so far. The rest of the paper is organized as follows. Section 2 reviews the literature and defines the need of our research work. Section 3 explains the system model, the analysis procedure and the proposed mathematical model. Section 4 details the ON model and Section 5 discusses the model performance. Section 6 concludes the paper.

\section{Procedure}

This paper insists the study on the degree to which the optical link nonlinearity gets impacted from the laser intensity. The system model is referred from [9]. Hence the input variables that are considered here include, the effective area (Aeff), the nonlinear regime of the transmitting fiber $\left(N_{1}\right)$ and the launch power $\left(P_{1}(\right.$ in $\left.\mathrm{dB})\right)$. The output variable under consideration is the bitwise error probability (BER), through which the effect of the input variables is studied. A system owning the configuration of Table 1 is employed and the BER versus the varying Optical Signal-to-Noise Ratio (OSNR) is yielded through different combinations of $N_{1}, A_{\text {eff }}$ and $P_{1}$. The range within which the values of $P_{1}, A_{\text {eff }}$ and $N_{1}$ get changed are, [-20 to $20 \mathrm{dBm}$ ], [60 to $100 \mu \mathrm{m} 2$ ] and [4.7x10-20-to $0.7 \times 10-20$ ], in order. A no cost optical communication toolbox, called as Optilux 0.1 , is made available from http://optilux.sourceforge.net/ to facilitate the simulation as well as to achieve further required analysis. From Fig. 1, we can observe that varying effective areas show very minute variation in the response of error probability with respect to the launch power. However, the variation in the nonlinear regime shows significant variations. When the nonlinear regime is minimum $(7 \times 10-21)$, a gradual decrease and increase in the error probability have been observed for varying launch power. The steep increase or decrease in the error probability has been observed for increasing nonlinear regime of the fiber. While performing the analysis, the actual (transmitted data pattern) and the received pattern (before thresholding) is observed to understand the nature of deviation with respect to the nonlinear regime. According to our previous results, the launch power has to be varied with respect to the nonlinear regime of the fiber to accomplish a desired error probability.

\subsection{Effective area from Schrodinger equation}

The existence of non-linearity in the optical fibers, the non-linear Schrodinger equation (NSE) has special importance in the propagation of light. However, from Maxwell's equation, the NSE equation has been derived. The name has been allotted to the equation because of the existence of the nonlinearity. Moreover, the energy packet illustrates that the particle kind of behavior. The amplitude encircles of the wave varies slowly in terms of the carrier (i.e.) the electric field is quasimonochromatic meaning fractional bandwidth of the wave is minimum. Moreover, this assumption is applicable for the majority of the communication signals since the modulation frequency of the order of $10^{9}-10^{10} \mathrm{~Hz}$ and the carrier frequency is of the order of $10^{10} \mathrm{~Hz}$.

The non-linearity is a small perturbation to the linear term.

- Throughout the propagation the polarization of the wave remains similar.

- Due to the non-linearity the transverse distribution of the modal fields is unaffected.

The electric field can be illustrated as

$$
E=M(s, \phi) A(z) e^{j(\omega t-\bar{\delta} z)}
$$

In Eq. (1) the cycle coordinate system is assumed and the model field distribution is $M(s, \phi)$ and the A $(z)$ denotes the slow varying encircle function of $z$. The model field distribution satisfies the linear wave by the Eq. (2)

$$
\nabla^{2} M+\left(\frac{\pi^{2} n^{2}}{c^{2}}-\delta^{2}\right) M=\nabla^{2} M+\left(\delta_{0}^{2}-\bar{\delta}_{0}^{2}\right) M=0
$$

In Eq. (2) the phase constant of the carrier in the optical fiber is $\delta_{0}$ and the model propagation constant is $\bar{\delta}$ and $\pi$ represents the angular frequency. Moreover, the Eq. (2) is same as the solved module on wave propagation inside an optical fiber. Assuming that the encircle function deviates slowly as a function of distance, the terms like $\frac{\partial^{2} A}{\partial z^{2}}$ are neglected and then the encircle function satisfies the Eq. (3)

$$
2 j \delta_{0} \frac{\partial A}{\partial z}+\left(\delta_{0}^{2}-\bar{\delta}_{0}^{2}\right) A=0
$$

From two special effects the propagation constant $\delta$ get contributes. First one occurs due to the dispersion (i.e.) it dependence on the frequency next because of the loss and non linear effects. Hence, it can be illustrated as Eq. (4)

$$
\bar{\delta}=\delta(\pi)+\Delta \delta
$$




$$
\begin{gathered}
\Delta \delta=\frac{\pi_{0}}{c} \frac{\int_{0}^{2 \omega \infty} \int_{0}^{\infty} \Delta n|M(s, \phi)|^{2} s d s d \phi}{\int_{0}^{2 \omega \infty} \int_{0}^{\infty}|M(s, \phi)|^{2} s d s d \phi} \\
\Delta n=n_{2}|E|^{2}-j \frac{\chi}{2} \frac{c}{\pi_{0}}
\end{gathered}
$$

In Eq. (6) the $\chi$ represents the attenuation constant of the fiber. Since, $\left(\bar{\delta}-\delta_{0}<\delta_{0}\right)$.This can approximate as $\left(\delta_{0}^{2}-\bar{\delta}^{2}\right) \approx 2 \delta_{0}\left(\delta_{0}-\bar{\delta}\right)$ and the Eq. (7) illustrates the encircle. Let us consider the Fourier transform of the Eq. (7) over time by the eq. (8) where, $\widetilde{A}$ is the Fourier transform of $A$ and it represented as Eq. (9)

$$
\begin{array}{r}
\frac{\partial A}{\partial z}-j\left(\delta_{0}-\delta(\pi)-\Delta \delta\right) A=0 \\
\frac{\partial A}{\partial z}-j\left(\delta_{0}-\delta(\pi)-\Delta \delta\right) \tilde{A}=0 \\
\tilde{A}\left(\pi-\pi_{0}\right)=\int_{-\infty}^{\infty} A(t) e^{-j\left(\pi-\pi_{0}\right) t} d t
\end{array}
$$

Then the inverse Fourier transform can be illustrated as Eq. (10) and the expansion of $\delta(\omega)$ in Taylor series around $\delta_{0}$ is defined in Eq. (11). In the envelop equation substitute the $\delta(\pi)$ and retaining the second derivatives terms of $\delta$ is given in the Eq.(13) and the Eq. (14) represents the encircle while taking inverse transform. In Non-Linear Schrodinger Equation (NSE) (14) the $\Delta \delta$ can be replace as Eq. (15).Then, the non linearity coefficient $\gamma$ can be represented as Eq.(16).Finally, the parameter $A_{\text {eff }}$ is represented as the effective area and it is illustrated as Eq. (17).

$$
\begin{gathered}
A(t)=\frac{1}{2 \omega} \int_{-\infty}^{\infty} \tilde{A}\left(\pi-\pi_{0}\right) e^{j\left(\pi-\pi_{0}\right) t} d \pi \\
\delta(\pi)=\delta_{0}+\left(\pi-\pi_{0}\right) \delta_{1}+\frac{1}{2}\left(\pi-\pi_{0}\right)^{2} \delta_{2}+\frac{1}{6}\left(\pi-\pi_{0}\right)^{3} \delta_{3}+\ldots \ldots \ldots \\
\delta_{n}=\left(\frac{\partial^{n} \delta}{\partial \pi^{n}}\right)_{\pi=\pi_{0}} \\
\frac{\partial \tilde{A}}{\partial z}+j\left(\pi-\pi_{0}\right) \delta_{1} \tilde{A}+j \frac{1}{2}\left(\pi-\pi_{0}\right) \delta_{2} \tilde{A}+j \Delta \delta \tilde{A}=0 \\
\frac{\partial A}{\partial z}+\delta_{1} \frac{\partial A}{\partial t}-j \frac{1}{2} \delta_{2} \frac{\partial^{2} A}{\partial t^{2}}+j \Delta \delta A=0 \\
\frac{\partial A}{\partial z}+\delta_{1} \frac{\partial A}{\partial t}-j \frac{1}{2} \delta_{2} \frac{\partial^{2} A}{\partial t^{2}}+\frac{\chi}{2} A+j \gamma\left|A^{2}\right| A=0
\end{gathered}
$$

$$
\begin{aligned}
& \gamma=\frac{n_{2} \pi_{0}}{c A_{\text {eff }}}\left.\int_{0 \text { eff }}^{2 \omega \infty} \int_{0}^{\infty}|M(s, \phi)|^{2} s d s d \phi\right)^{2} \\
& \int_{0}^{2 \omega \infty} \int_{0}^{\infty}|M(s, \phi)|^{4} s d s d \phi
\end{aligned}
$$

\section{Optical-neural model based on time series forecasting}

The simulation of the optical link is accomplished with the help of the optical neural model, which utilizes BER, $A_{e f f}, P_{l}$ and $N_{l}$. This implies that the suggested optical - neural model effectively performs the estimation of the probable $P_{l}$, once it is provided with the desired BER, $A_{\text {eff }}$ and $N_{l}$. It monitors the previous investigation results for adapting on its own and to make a response in accordance. Our earlier model has been inspired from feed forward neural network by Levenberg Marquardt algorithm [10,11]. However, the algorithm is not robust against considering more number of optical link inputs. Hence this paper adopts nonlinear autoregressive model with exogenous input, often termed as NARX neural network.

NARX model constitute the chief class, which comes under the category of discrete-time nonlinear systems $\quad[12-16], \quad y(t)=f\left[u\left(t-D_{u}\right), \ldots, u(t-1) \cdot u(t) . y(t-\right.$ $\left.\left.D_{y}\right), \ldots y(t-1)\right]$ where, $u(t)$ denotes the network's input and $y(t)$ specifies the network's output, when the time is $t$. The input order and the output order are orderly represented as $D_{u}$ and $D_{y}$. Here, $f$ stands for the nonlinear function. This nonlinear function $(f)$ when subjected to approximation using a Multilayer Perceptron (MLP), would cause the emergence of a system, termed as, NARX recurrent neural network $[12,17]$.

This paper deals with a complete examination of the NARX networks, which own an input order of zero and the output dimension as one. This implies the networks, wherein the output alone gives rise to feedback. Yet, the reason for the inability to extend our results using networks of higher input orders could not precisely stated. Hence, the expression that defines the functioning of the NARX networks with an input order of zero is $y(t)=\Psi[u(t), y(t$ 1)...y $(t-D)]$, where, the function $\Psi$ denotes the mapping that is established through the MLP as shown in Fig. 2. The weight links that are portrayed in Fig. 2 may be subjected to changes or maintained constant. It is the application that sets those weight links as constant or adaptable. 


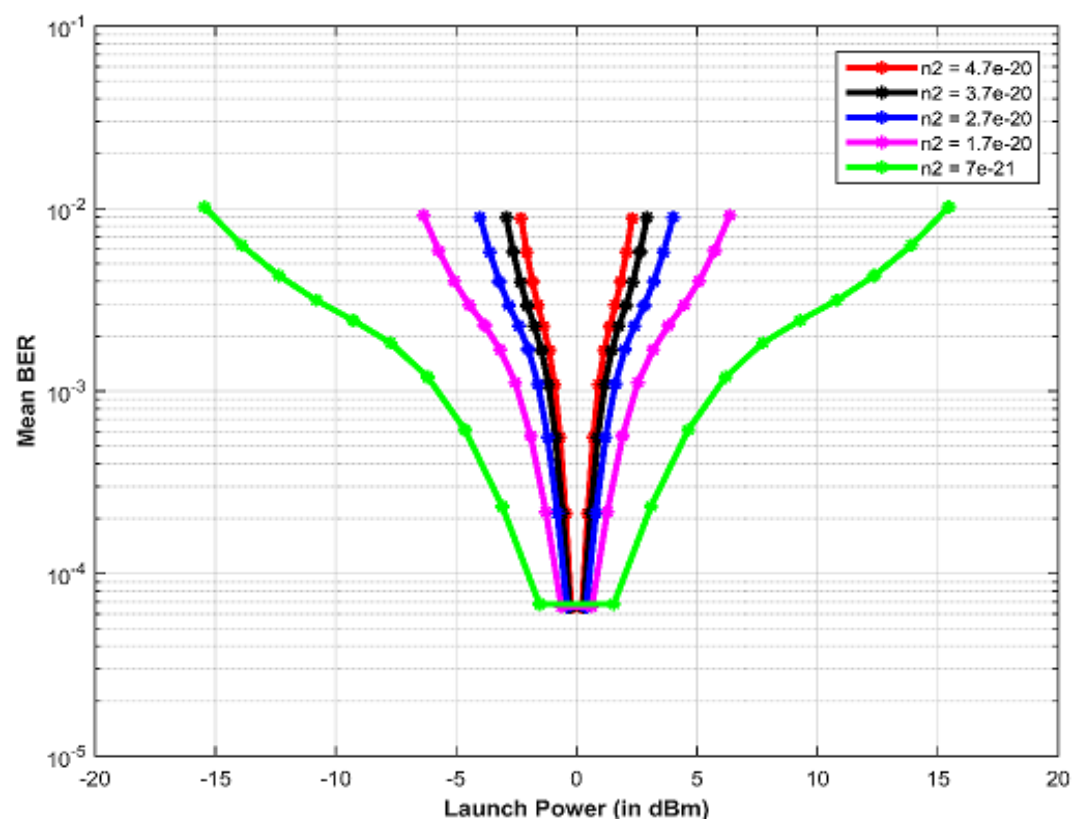

Figure. 1 Impact of nonlinear regime and launch power on the error probability of the optical link for effective area of 60 $\mathrm{m}^{2}$

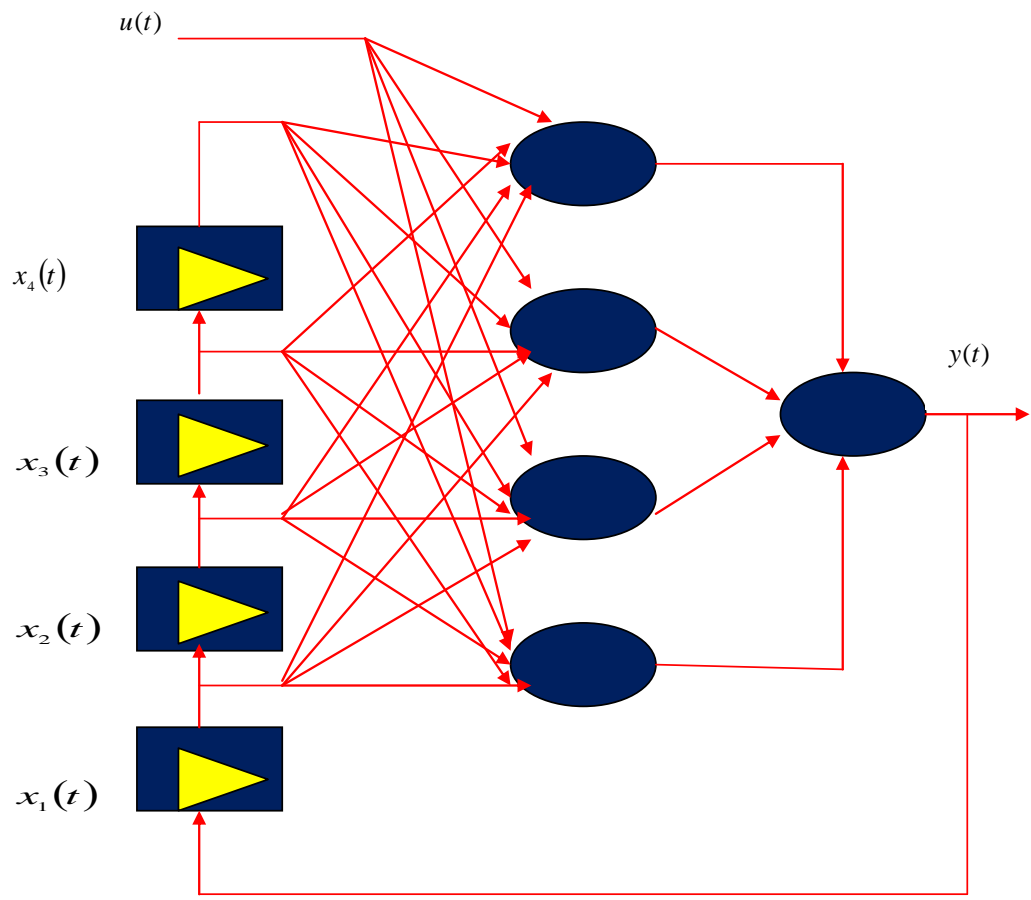

Figure. 2 The architecture of NARX used to simulate the optical link

Representing the equations in the state-space form is more suitable in view point of the system [18]. This representation form allows the examination as well as the derivation of the Jacobian [19]. Usually, when realizing the discrete-time dynamical system, unit-delay elements have linkage to the states of the system always. Therefore, the state-space representation of that sort of systems can be specified as:

$$
x_{i}(t+1)=\left\{\begin{array}{cc}
\Psi[u(t), X(t)] & i=1 \\
x_{i-1}(t) & i=2, \ldots, D
\end{array}\right.
$$

$$
y(t)=x_{1}(t+1)
$$

The NARX networks do not exhibit a high level of resistance against the long-term dependency issue. For the given state-space map, the Jacobian matrix is given as,

$$
\begin{gathered}
J_{X}(t+1,1)=\nabla_{X(t)} X(t+1) \\
J_{X}(t+1,1)=\left[\begin{array}{ccccc}
\frac{\partial \Psi(t)}{\partial x_{1}(t)} & \frac{\partial \Psi(t)}{\partial x_{2}(t)} & \cdots & \frac{\partial \Psi(t)}{\partial x_{D-1}(t)} & \frac{\partial \Psi(t)}{\partial x_{D}(t)} \\
1 & 0 & \ldots & 0 & 0 \\
0 & 1 & \ldots & 0 & 0 \\
\cdots & \ldots & \ddots & \ldots & \ldots \\
0 & 0 & \ldots & 1 & 0
\end{array}\right]
\end{gathered}
$$


The complete Eigen values of the Jacobian that were obtained at every single time step, when found to lie within a circle of unit radius, indicate that the network's states would belong to the reduced attracting set of any one of the hyperbolic attractors. This, in turn, denotes the latching of the system at that moment with increased robustness. This again specifies $\lim _{n \rightarrow \infty} J_{X(t, n)}=0$, which is similar to all the remaining recurrent neural networks. However, for the sake of simplicity, this paper exploits the Levenberg - Marquardt training [11] explained in our previous paper [9].

\section{Results and discussion}

\subsection{Experimental setup}

The proposed optical model is simulated in MATLAB R2014a and experimented in a 64-bit computing machine with i5 processor, 8GB RAM and windows OS. The performance investigation is carried out by defining the desired BER as $10^{-7}$ and the nonlinear regime and the effective area are varied from $\left[5 \times 10^{-20}, 1 \times 10^{-20}\right]$ and $75 \mathrm{~m}^{2}$ and $85 \mathrm{~m}^{2}$, respectively. For such different input variations, the launch power is estimated and it is subjected to the optical link of Optilux tool. Hence obtained BER is compared with the desired BER for the deviation and so the error absolute has been calculated. Based on the obtained error absolute for different experimental scenario, the further performance investigations are carried out.

\subsection{Impact of model architecture}

The architecture of neural model plays crucial role in determining the precision of the proposed modeling technique. The architecture/ configuration of the neural model have been varied by changing the number of hidden neurons. The analysis includes experimenting on two variants of network architectures, single layer and double layer. In the single layer network architecture, there is only one hidden layer, which has been varied from 10 neurons to 90 neurons with an increasing rate of 10 neurons. Hence, nine degree of experiments has been obtained to observe the impact of the configuration of the network. Similarly, the double layer network, which has two hidden layers, has also obtained nine variants of configuration, but here, each hidden layer has a variety of 10, 20 and 30 neurons and hence the configuration gets their combination as mentioned in Fig. 3 and Fig. 4.

According to Fig. 3 and 4, the single layer neural model has to be constructed simple enough, i.e. with
10 or 20 hidden neurons, to ensure fair performance, because they exhibit relatively minimum error compared with the model with more number of neurons. The similar results can be observed from Fig. 4 and 5. In the case of double layer neural model, a more interesting outcome has been observed. The Fig. 4 and Fig. 5 demonstrate that the minimum absolute error can be achieved only if both the hidden neurons are balanced, i.e. the number of hidden neurons in both the layers must be closer to each other, especially in the configuration $(30,20)$. Moreover, they do not prefer high degree of configuration such as $(50,50)$, which shows that the optical link prefers less complex architecture for its characteristics.

Table 1 and 2 compare the proposed method with the three existing methods under the single and multi layer neural models. The methods adopted for comparison are adaptive Levenberg-Marquardt algorithm (aLM) [20], hybrid Genetic Algorithm (hGA) [21] and GbLN PSO algorithm (GPSO) [22]. As is seen in Table 1, the single layer the proposed method gives the first best result while comparing with the existing algorithms. In Table 2, the proposed algorithm achieves second best result after the Evolutionary method but in $N_{l}=4 \times 10^{-20}$ and $N_{l}=$ $3 \times 10^{-20}$, the proposed algorithm achieves first best result than the existing algorithm. From the tables; it is clearly evident that the proposed algorithm achieves better performance than the existing algorithms.

\subsection{Impact of nonlinear regime of the fiber}

The impact of nonlinear regime on the fiber has been demonstrated through Fig. 3. It plays a major role in producing systematic performance from single layer network architecture. According to Fig. 3 (a) and (b) the network architecture with minimum configuration can produce least absolute error for all the nonlinear regimes. The absolute error has been gradually increasing with respect to increasing the complexity of the network architecture. Though such proportional relationship can be seen in the early network architectures, the higher stages of network complexity such as network with 40, 50, 60, 70, 80 and 90 neurons could not exhibit such relationships. Moreover, such high complex single layer architectures produce poor error minimization performance. Hence, the single layer network with more number of hidden neurons is not viable for the proposed optical - neural model.

On the other hand, Fig. 3 (c) and (d) exhibits the impact of nonlinear regime of the fiber through double layer network architecture. In contrast to the 
single layer architecture, the double layer architecture neural network does not exhibit relationship with the nonlinear regime of the fiber. In other words, nonlinear regime of the fiber is not sensitive to the architecture of the neural network. The simulated optical link correlates well for lesser nonlinear regime out of the five nonlinear regime variations, when experimenting for both the effective areas.

\subsection{Impact of effective area of the fiber}

The impact of effective area of the simulated model of the optical link is demonstrated in Fig. 4 and 5. The effective area has good correlation with the optical link, because it exhibits minimal absolute error for simple network configuration, though the

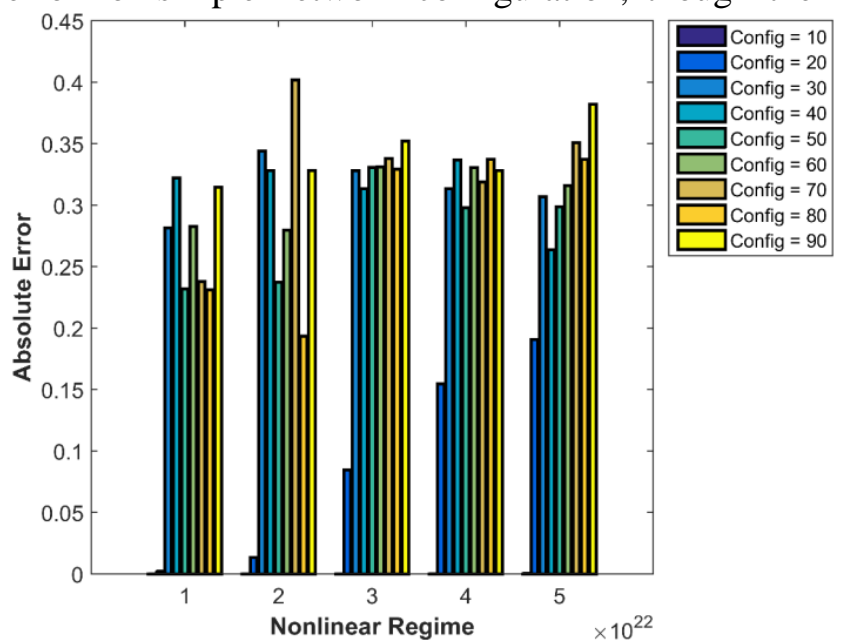

(a)

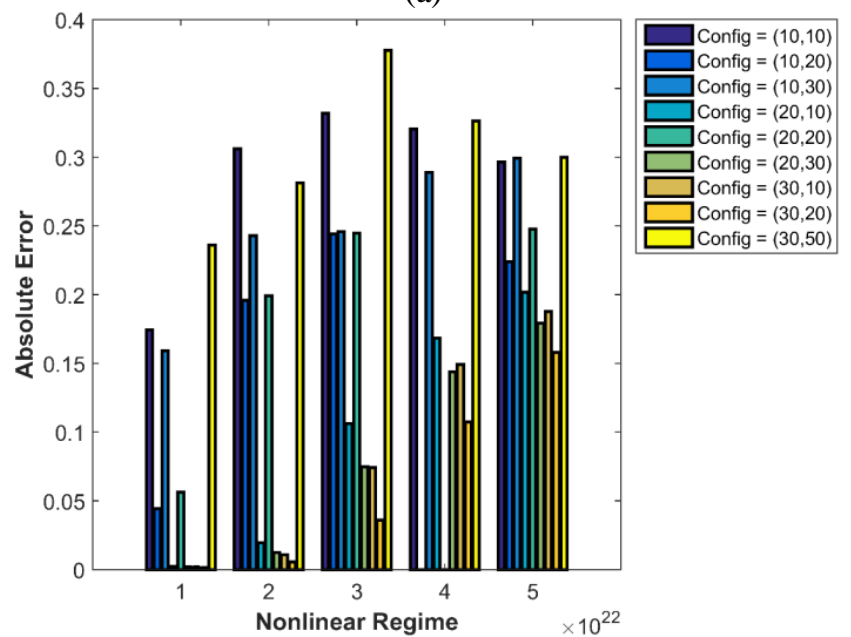

(c) nonlinear regime of the fiber has been varied. Such metric association could be found in Fig. 4, where single layer network architecture has been considered. In addition with the information, the Fig. 4 demonstrates that the maximum absolute error that has been attained for both the effective area remains lesser than 0.4. The similar kind of error absolution can be experienced from Fig. 5, where the double layer network has been used for the model. The contextual difference between Fig. 4 and Fig. 5 is that the single layer architecture produces systematic absolute error accomplishment, whereas the double layer architecture is random in accomplishing the said performance. The impact of the network architecture has been already discussed in this Section.

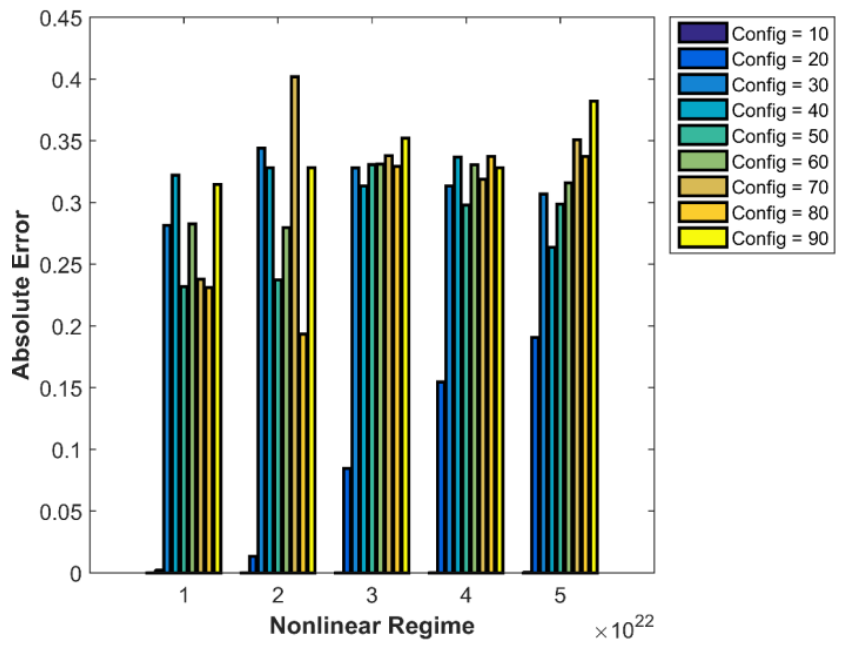

(b)

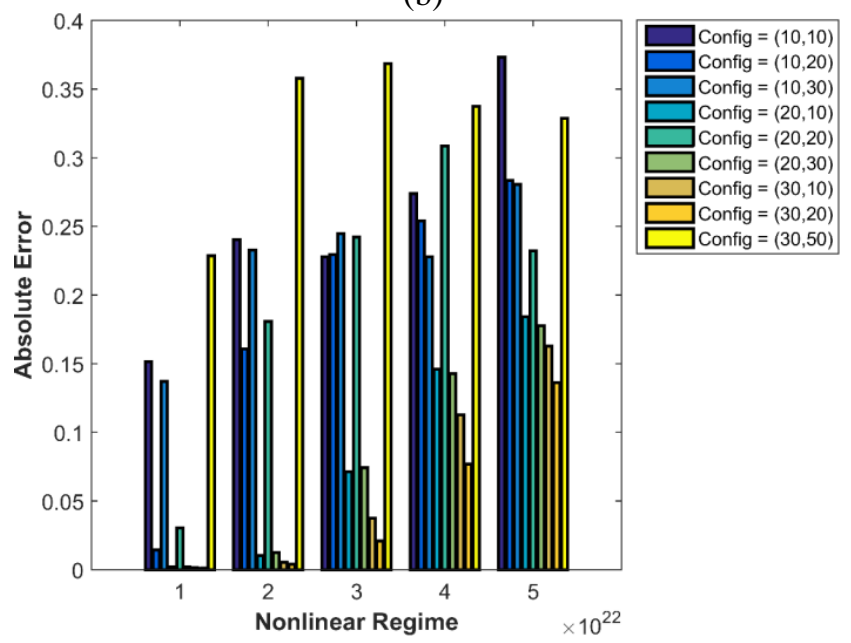

(d)

Figure. 3 Error absolute with respect to varying nonlinear regime, when (a) single layer NARX model is experimented on effective area of $75 \mathrm{~m}^{2}$ and (b) $85 \mathrm{~m}^{2}$ and (c) and (d) are for double layer NARX model experimented on the same two effective areas, respectively 

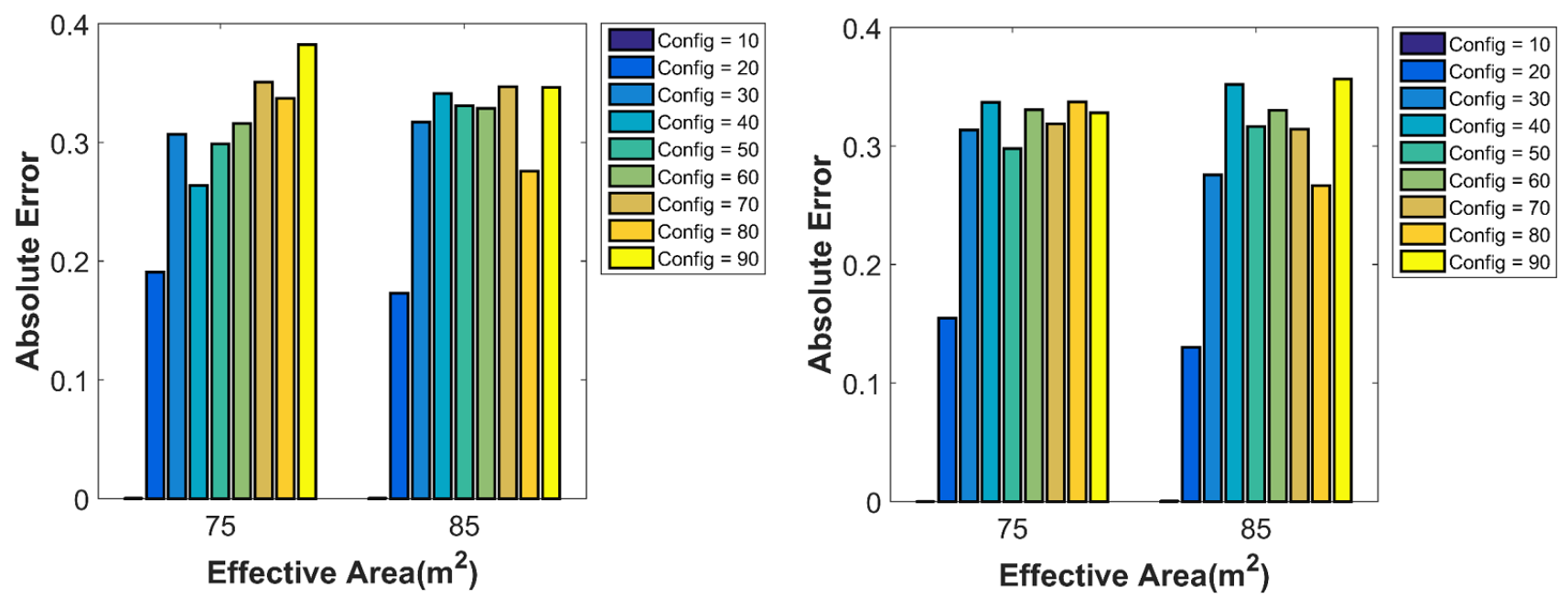

Effective Area $\left(\mathrm{m}^{2}\right)$

(a)

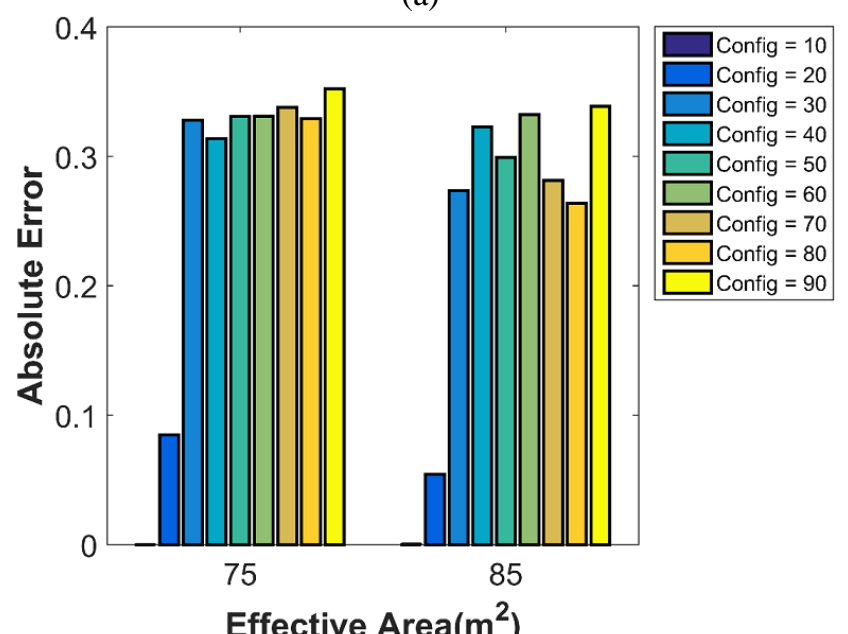

(c)
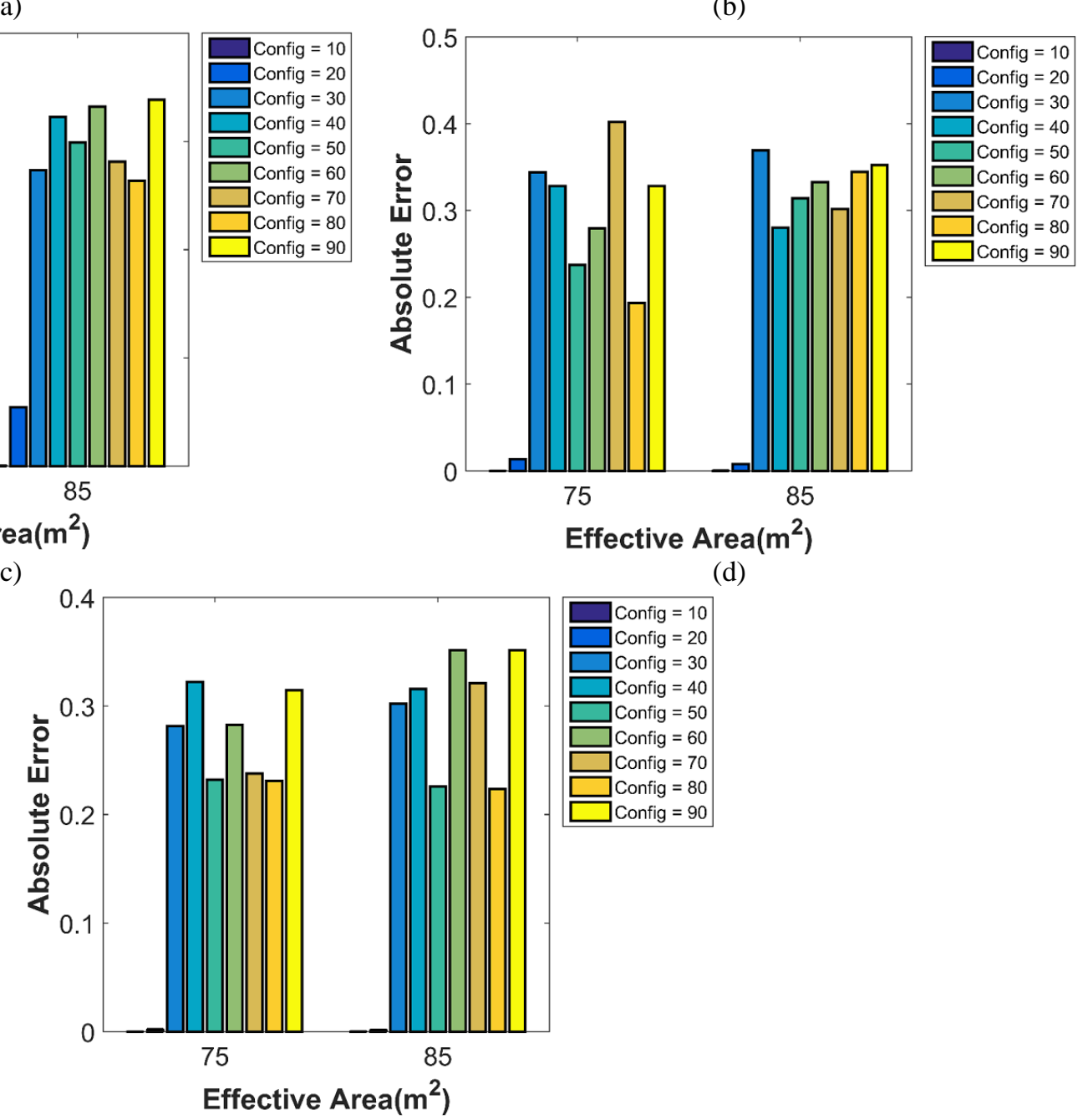

(e)

Figure. 4 Error absolute obtained from single layer NARX model for varying number of hidden neurons and effective area, when the nonlinear regime is set as (a) $5 \times 10^{-20}$, (b) $4 \times 10^{-20}$, (c) $3 \times 10^{-20}$, (d) $2 \times 10^{-20}$ and (e) $1 \times 10^{-20}$. 

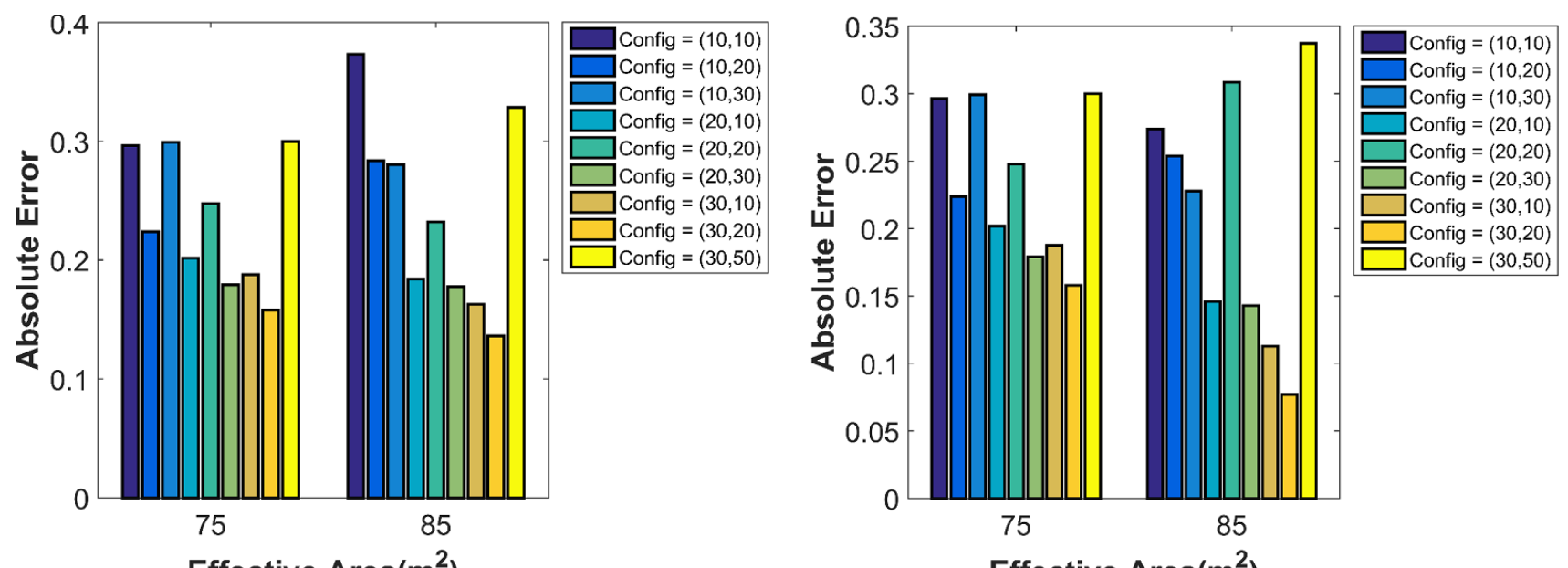

Effective Area $\left(\mathrm{m}^{2}\right)$

(a)

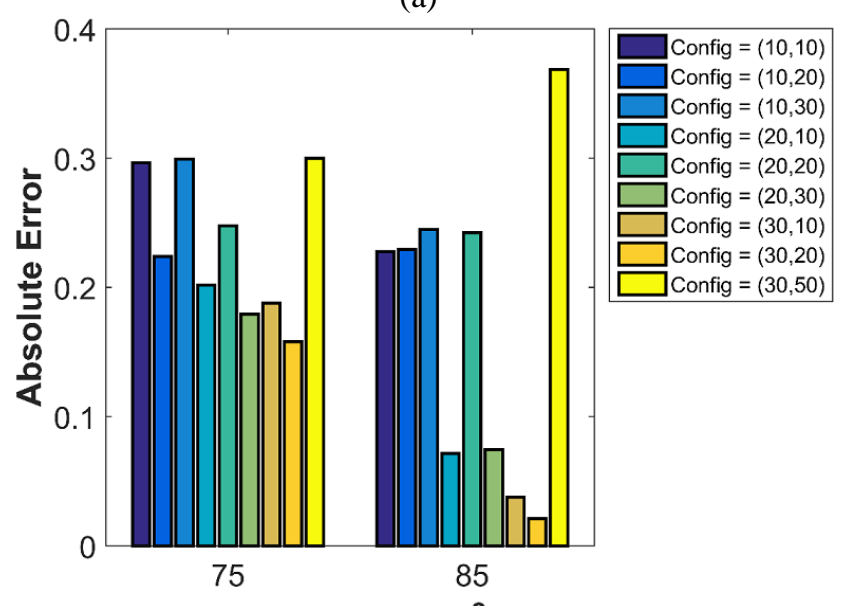

Effective Area $\left(\mathrm{m}^{2}\right)$

(c)

Effective Area $\left(\mathrm{m}^{2}\right)$

(b)

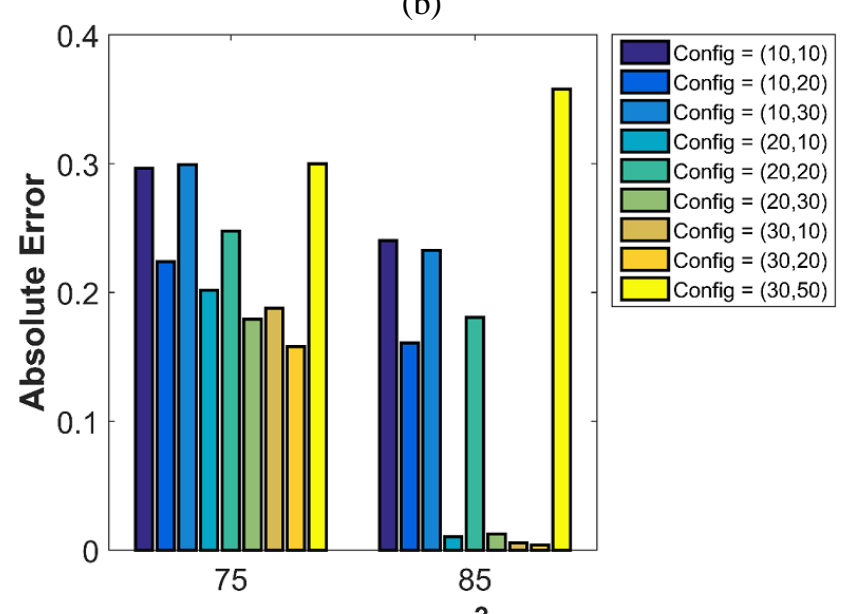

Effective Area $\left(\mathrm{m}^{2}\right)$

(d)

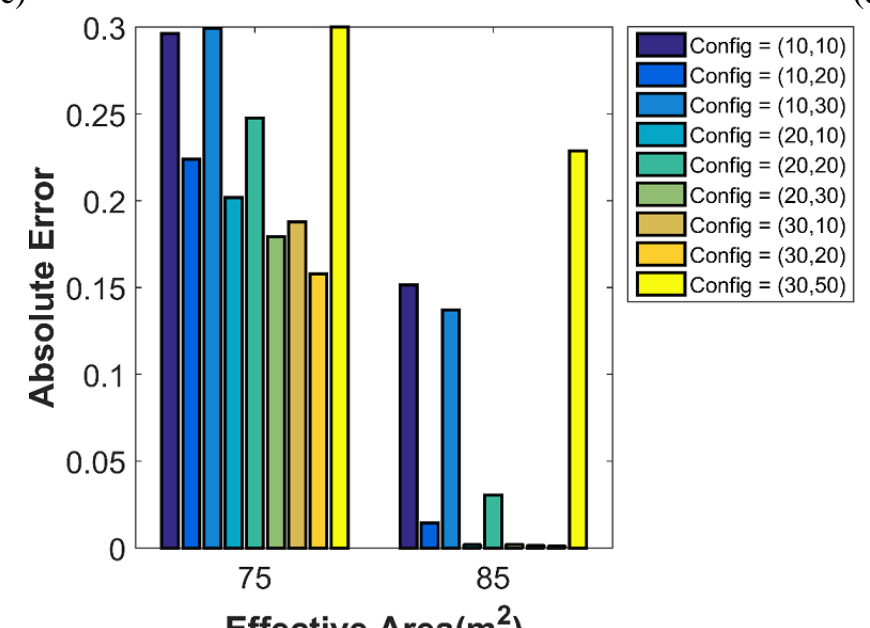

Effective Area $\left(\mathrm{m}^{2}\right)$

(e)

Figure. 5 Error absolute obtained from double layer NARX model for varying number of hidden neurons and effective area, when the nonlinear regime is set as (a) $5 \times 10^{-20}$, (b) $4 \times 10^{-20}$, (c) $3 \times 10^{-20}$, (d) $2 \times 10^{-20}$ and (e) $1 \times 10^{-20}$ 
Table 1. Comparison of error absolute obtained from Single layer between the existing and proposed method

\begin{tabular}{|c|c|c|c|c|c|c|c|c|c|c|c|c|}
\hline \multicolumn{2}{|c|}{ Degree of the layer } & 10 & 20 & 30 & 40 & 50 & 60 & 70 & 80 & 90 & $\begin{array}{c}\text { Average Error } \\
\text { Absolute }\end{array}$ & $\begin{array}{l}\text { Final } \\
\text { Rank }\end{array}$ \\
\hline \multirow{4}{*}{$\begin{array}{c}N_{l}= \\
5 \times 10^{-20}\end{array}$} & Proposed & $0.00(1)$ & $0.16(1)$ & $0.20(2)$ & $0.28(1)$ & $0.25(1)$ & $0.38(4)$ & $0.35(2)$ & $0.34(3)$ & $0.25(1)$ & 1.78 & 1 \\
\hline & aLM [20] & $0.09(2)$ & $0.32(3)$ & $0.17(1)$ & $0.35(3)$ & $0.37(4)$ & $0.21(1)$ & $0.38(4)$ & $0.39(4)$ & $0.39(4)$ & 2.89 & 4 \\
\hline & hGA [21] & $0.32(4)$ & $0.36(4)$ & $0.37(4)$ & $0.36(4)$ & $0.31(2)$ & $0.35(3)$ & $0.09(1)$ & $0.33(1)$ & $0.29(2)$ & 2.78 & 3 \\
\hline & GPSO [22] & $0.31(3)$ & $0.25(2)$ & $0.33(3)$ & $0.33(2)$ & $0.33(3)$ & $0.32(2)$ & $0.37(3)$ & $0.33(2)$ & $0.34(3)$ & 2.56 & 2 \\
\hline \multirow{4}{*}{$\begin{array}{c}N_{l}= \\
4 \times 10^{-20}\end{array}$} & Proposed & $0.00(1)$ & $0.11(1)$ & $0.16(2)$ & $0.37(4)$ & $0.26(2)$ & $0.32(4)$ & $0.31(2)$ & $0.30(1)$ & $0.19(1)$ & 2 & 1 \\
\hline & \begin{tabular}{|ll} 
aLM $[20]$ \\
\end{tabular} & $0.04(2)$ & $0.38(4)$ & $0.13(1)$ & $0.36(3)$ & $0.35(4)$ & $0.19(1)$ & $0.39(4)$ & $0.39(4)$ & $0.38(3)$ & 2.89 & 4 \\
\hline & hGA [21] & $0.29(3)$ & $0.33(3)$ & $0.35(4)$ & $0.32(2)$ & $0.25(1)$ & $0.32(3)$ & $0.04(1)$ & $0.37(3)$ & $0.39(4)$ & 2.67 & 3 \\
\hline & GPSO [22] & $0.34(4)$ & $0.24(2)$ & $0.31(3)$ & $0.29(1)$ & $0.32(3)$ & $0.30(2)$ & $0.31(3)$ & $0.35(2)$ & $0.25(2)$ & 2.44 & 2 \\
\hline \multirow{4}{*}{$\begin{array}{c}N_{l}= \\
3 \times 10^{-20}\end{array}$} & Proposed & $0.08(1)$ & $0.04(1)$ & $0.09(2)$ & $0.28(2)$ & $0.31(3)$ & $0.30(2)$ & $0.32(2)$ & $0.29(1)$ & $0.04(1)$ & 1.67 & 1 \\
\hline & aLM [20] & $0.01(2)$ & $0.36(4)$ & $0.05(1)$ & $0.38(4)$ & $0.39(4)$ & $0.14(1)$ & $0.38(3)$ & $0.34(3)$ & $0.38(4)$ & 2.89 & 4 \\
\hline & hGA [21] & $0.31(4)$ & $0.32(3)$ & $0.35(4)$ & $0.25(1)$ & $0.23(1)$ & $0.40(4)$ & $0.00(1)$ & $0.35(4)$ & $0.28(3)$ & 2.78 & 3 \\
\hline & GPSO [22] & $0.28(3)$ & $0.19(2)$ & $0.29(3)$ & $0.32(3)$ & $0.25(2)$ & $0.32(3)$ & $0.38(4)$ & $0.30(2)$ & $0.23(2)$ & 2.67 & 2 \\
\hline \multirow{4}{*}{$\begin{array}{c}N_{l}= \\
2 \times 10^{-20}\end{array}$} & Proposed & $0.00(1)$ & $0.00(1)$ & $0.01(2)$ & $0.30(3)$ & $0.23(2)$ & $0.35(4)$ & $0.31(2)$ & $0.32(2)$ & $0.00(1)$ & 2 & 1 \\
\hline & \begin{tabular}{|ll}
$\mathrm{aLM}$ & {$[\mathbf{2 0}]$} \\
\end{tabular} & $0.02(2)$ & $0.31(3)$ & $0.07(1)$ & $0.36(4)$ & $0.38(4)$ & $0.04(1)$ & $0.40(4)$ & $0.36(4)$ & $0.34(4)$ & 3 & 4 \\
\hline & hGA [21] & $0.24(3)$ & $0.31(4)$ & $0.38(4)$ & $0.23(1)$ & $0.16(1)$ & $0.31(3)$ & $0.00(1)$ & $0.32(3)$ & $0.24(2)$ & 2.44 & 2 \\
\hline & G PSO[22] & $0.34(4)$ & $0.11(2)$ & $0.25(3)$ & $0.26(2)$ & $0.35(3)$ & $0.28(2)$ & \begin{tabular}{|l|}
$0.34(3)$ \\
\end{tabular} & $0.31(1)$ & $0.24(3)$ & 2.56 & 3 \\
\hline \multirow{4}{*}{$\begin{array}{c}N_{l}= \\
1 \times 10^{-20}\end{array}$} & \begin{tabular}{|l|} 
Proposed \\
\end{tabular} & 5.9E05(1) & $0.00(1)$ & $0.02(2)$ & \begin{tabular}{|l|}
$0.24(2)$ \\
\end{tabular} & \begin{tabular}{|l|}
$0.11(2)$ \\
\end{tabular} & \begin{tabular}{|l|}
$0.36(4)$ \\
\end{tabular} & $0.33(3)$ & $0.19(1)$ & $2.0 \mathrm{E} 05(1)$ & 1.89 & 1 \\
\hline & $\begin{array}{|ll|}\text { aLM } & {[20]} \\
\end{array}$ & $0.08(2)$ & $0.30(4)$ & $0.02(1)$ & $0.31(4)$ & $\mid 0.37(4)$ & $0.00(1)$ & \begin{tabular}{|l|}
$0.39(4)$ \\
\end{tabular} & $0.32(4)$ & $0.37(4)$ & 3.11 & 4 \\
\hline & hGA [21] & $0.05(3)$ & $0.24(3)$ & $0.35(4)$ & 0.31(3) & $0.02(1)$ & $0.32(2)$ & $0.07(1)$ & $0.24(3)$ & $0.19(3)$ & 2.56 & 3 \\
\hline & GPSO [22] & $0.32(4)$ & $0.06(2)$ & $0.24(3)$ & $0.17(1)$ & $0.25(3)$ & $0.32(3)$ & $0.26(2)$ & $0.19(2)$ & $0.15(2)$ & 2.44 & 2 \\
\hline
\end{tabular}

Table 2. Comparison of error absolute obtained from Double layer between the existing and proposed method

\begin{tabular}{|c|c|c|c|c|c|c|c|c|c|c|c|}
\hline \begin{tabular}{|l}
$\begin{array}{c}\text { Degree } \\
\text { of the } \\
\text { layer }\end{array}$ \\
\end{tabular} & $(10,10)$ & $(10,20)$ & $(10,30)$ & $(20,10)$ & $(20,20)$ & $(20,30)$ & $(30,10)$ & $(30,20)$ & $(30,30)$ & $\begin{array}{l}\text { Average } \\
\text { Error } \\
\text { Absolute } \\
\end{array}$ & $\begin{array}{c}\text { Average } \\
\text { Rank } \\
\text { (FinalRank) } \\
\end{array}$ \\
\hline \multirow{4}{*}{$\begin{array}{c}N_{l}= \\
5 \times 10^{-20}\end{array}$} & Proposed & $0.09(3)$ & $0.24(1)$ & $0.21(1)$ & $0.21(1)$ & $0.26(2)$ & $0.21(1)$ & $0.26(3)$ & $0.11(2)$ & \begin{tabular}{|l|}
$0.38(4)$ \\
\end{tabular} & 2 \\
\hline & aLM [20] & $0.38(4)$ & $0.33(4)$ & $0.39(4)$ & $0.38(4)$ & $0.32(4)$ & $0.35(4)$ & $0.40(4)$ & $0.35(4)$ & \begin{tabular}{|l|}
$0.36(3)$ \\
\end{tabular} & 4 \\
\hline & hGA [21] & $0.01(1)$ & $0.32(3)$ & $0.21(2)$ & $0.29(2)$ & $0.07(1)$ & $0.38(2)$ & $0.01(2)$ & $4.0 \mathrm{e} 05(1)$ & $0.29(1)$ & 1 \\
\hline & GPSO[22] & $0.01(2)$ & $0.30(2)$ & $0.31(3)$ & $0.331(3)$ & $0.32(3)$ & $0.34(3)$ & $0.03(1)$ & $0.31(3)$ & $0.31(2)$ & 2 \\
\hline \multirow{4}{*}{$\begin{array}{c}N_{l}= \\
\mathbf{4 \times 1 0 ^ { - 2 0 }}\end{array}$} & Proposed & $0.03(3)$ & $0.25(1)$ & $0.18(1)$ & $0.172(1)$ & $0.25(3)$ & $0.18(1)$ & $0.32(3)$ & $0.06(2)$ & $0.32(2)$ & 1 \\
\hline & aLM [20] & $0.38(4)$ & $0.32(2)$ & $0.31(4)$ & $0.39(4)$ & $0.33(4)$ & $0.32(4)$ & $0.35(4)$ & $0.37(4)$ & $0.39(4)$ & 4 \\
\hline & $(\mathrm{hGA})[21]$ & $0.006(1)$ & $0.38(4)$ & $0.28(3)$ & $0.36(3)$ & $0.04(1)$ & $0.32(3)$ & $0.05(2)$ & 3.1E05(1) & $0.37(3)$ & 2 \\
\hline & GPSO[22] & $0.04(2)$ & $0.33(3)$ & $0.24(2)$ & $0.32(2)$ & $0.23(2)$ & $0.24(2)$ & $0.01(1)$ & $0.33(3)$ & $0.25(1)$ & 2 \\
\hline \multirow{4}{*}{$\begin{array}{c}N_{I}= \\
3 \times 10^{-20}\end{array}$} & Proposed & $0.08(3)$ & $0.20(1)$ & $0.13(1)$ & $0.11(1)$ & $0.22(2)$ & $0.12(1)$ & $0.24(3)$ & $0.02(2)$ & $0.31(3)$ & 1 \\
\hline & aLM [20] & $0.37(4)$ & $0.355(4)$ & $0.32(4)$ & \begin{tabular}{|l|}
$0.39(4)$ \\
\end{tabular} & $0.30(4)$ & $0.31(2)$ & $0.36(4)$ & $0.35(4)$ & \begin{tabular}{|l|}
$0.34(4)$ \\
\end{tabular} & 4 \\
\hline & hGA [21] & $0.003(1)$ & $0.31(2)$ & $0.31(3)$ & $0.35(3)$ & $0.02(1)$ & $0.35(4)$ & $0.02(2)$ & $2.405(1)$ & $0.25(2)$ & 2 \\
\hline & GPSO[22] & $0.02(2)$ & $0.35(3)$ & $0.24(2)$ & $0.33(2)$ & $0.26(3)$ & $0.33(3)$ & $0.01(1)$ & $0.31(3)$ & $0.23(1)$ & 2 \\
\hline \multirow{4}{*}{$\begin{array}{c}N_{I}= \\
2 \times 10^{-20}\end{array}$} & Proposed & $0.03(3)$ & $0.14(1)$ & $0.03(1)$ & $0.02(1)$ & $0.16(2)$ & $0.03(1)$ & $0.19(3)$ & $0.04(2)$ & \begin{tabular}{|l|}
$0.37(4)$ \\
\end{tabular} & 2 \\
\hline & \begin{tabular}{|l|} 
aLM [20] \\
\end{tabular} & $0.36(4)$ & $0.22(2)$ & $0.26(4)$ & \begin{tabular}{|l|}
$0.33(3)$ \\
\end{tabular} & $0.34(4)$ & $0.28(2)$ & $0.32(4)$ & $0.32(3)$ & \begin{tabular}{|l|}
$0.33(3)$ \\
\end{tabular} & 4 \\
\hline & \begin{tabular}{|l|} 
hGA [21] \\
\end{tabular} & $0.001(1)$ & $0.23(3)$ & $0.24(2)$ & \begin{tabular}{|l|}
$0.37(4)$ \\
\end{tabular} & $8.9 \mathrm{E}-05(1)$ & $0.32(3)$ & $0.01(2)$ & $1.8 \mathrm{E}-05(1)$ & \begin{tabular}{|l|}
$0.28(2)$ \\
\end{tabular} & 2 \\
\hline & GPSO[22] & $0.01(2)$ & $0.37(4)$ & $0.24(3)$ & $0.31(2)$ & $0.21(3)$ & $0.33(4)$ & $0.004(1)$ & $0.36(4)$ & $0.16(1)$ & 3 \\
\hline \multirow{4}{*}{$\begin{array}{c}N_{l}= \\
1 \times 10^{-20}\end{array}$} & Proposed & $0.07(3)$ & $0.09(1)$ & $0.00(1)$ & $0.02(1)$ & $0.01(2)$ & $0.03(2)$ & $0.03(3)$ & $0.01(2)$ & $0.23(3)$ & 2 \\
\hline & aLM [20] & $0.31(4)$ & $0.24(4)$ & $0.17(4)$ & $0.36(4)$ & $0.23(4)$ & $0.35(4)$ & $0.28(4)$ & $0.35(4)$ & \begin{tabular}{|l|}
$0.39(4)$ \\
\end{tabular} & 4 \\
\hline & hGA [21] & 4.3E-05(1) & $0.02(2)$ & $0.13(2)$ & $0.24(2)$ & 3.4E-05(1) & $8.1 \mathrm{E}-05(1)$ & $0.02(2)$ & $1.4 \mathrm{E}-05(1)$ & \begin{tabular}{|l|}
$0.09(2)$ \\
\end{tabular} & 1 \\
\hline & GPSO[22] & $0.02(2)$ & $0.24(3)$ & $0.16(3)$ & \begin{tabular}{|l|}
$0.28(3)$ \\
\end{tabular} & $0.08(3)$ & $0.20(3)$ & 9.9E-05(1) & $0.24(3)$ & \begin{tabular}{|l|}
$0.02(1)$ \\
\end{tabular} & 2 \\
\hline
\end{tabular}

\section{Conclusion}

This paper extended our previous analysis and modeling method for optical link to estimate the required launch power under varying nonlinear regime of the fiber to meet the desired BER. The improvement our previous work has been accomplished through extending the nonlinear analysis by varying the effective area of the fiber. Though the nonlinear impact exhibited by the effective area is small on the estimation process, it should be considered because of the significance of every minor variation in the error probability. In order to handle multiple nonlinear parameters, we exploit the NARX neural model to simulate optical link. The modeling performance has been observed under varying experimental scenarios from which the precision of the proposed model has been ensured. The experimental results show that the proposed model is efficient and it achieves the first rank for four times in single layer neural network. However, in double layer neural network, the proposed model achieves the first rank twice. Hence, the overall performance of the proposed model is found to be better than the existing method, through the absolute error evaluation on the nonlinear 
regime of the fiber properties. Since the model outcome highly correlate with the characteristics of the optical link, an intelligent toolbox will be derived based on the proposed neural models. The neural model substantially reduces the experimentation cost and so better communication link can be established for improved system performance.

\section{References}

[1] M. Sung, S. Kang, J. Shim, J. Lee, and J. Jeong, "DFT-Precoded Coherent Optical OFDM with Hermitian Symmetry for Fiber Nonlinearity Mitigation", Journal of Light wave Technology, Vol.30, No.17, pp.2757 - 2763, 2012.

[2] H.Wang, Y.Li, X.Yi, D.Kong, J.Wu, and J.Lin, "APSK Modulated CO-OFDM System With Increased Tolerance Towards Fiber Nonlinearity", Photonics Technology Letters, IEEE, Vol.24, No.13, pp.1085 - 1087, 2012.

[3] E.B.Desurvire, "Capacity demand and technology challenges for light wave systems in the next two decades", Journal of Light wave Technology, Vol.24,No.12, pp.4697-4710, 2006.

[4] R. J. Essiambre, G. Kramer, P. J. Winzer, G. J. Foschini, and B. Goebel, "Capacity limits of optical fiber networks", Journal of Light wave Technology, Vol.28, No.4, pp.662-701, 2010.

[5] X. Chen, and W. Shieh, "Closed-form expressions for nonlinear transmission performance of densely spaced coherent optical OFDM systems", Opt. Exp., Vol.18, No.18, pp.19039-19054, 2010.

[6] W. Shieh, and X. Chen, "Information spectral efficiency and launch power density limits due to fiber nonlinearity for coherent optical OFDM systems", IEEE, Photon. Journal, Vol.3, No.2, pp.158-173, 2011.

[7] S.L.Jansen, D.Borne, P.M.Krummrich, S. Spalter, G.D.Khoe, and H.Waardt, "Longhaul DWDM transmission systems employing optical phase conjugation", IEEE Journal of Selected Topics in Quantum Electronics, Vol.12, No.4, pp.505-520, 2006.

[8] Y.Kim, S.Ju, S. Jeong, J-Y. Kim, N-H. Lee, H-K. Jung, and W-T. Han, "Gamma-Ray Radiation Effect on Non-Resonant Third-Order Optical Nonlinearity of Germano-Silicate Optical Fiber", Nuclear Science, IEEE Transactions, Vol.62, No.3, pp.1362 - 1366, 2015.

[9] K.Bhatnagar and S.C. Gupta, "Investigating and Modeling the Effect of Laser Intensity and Nonlinear Regime of the Fiber on Optical Link", Journal of Optical Communications.
[10] K.Levenberg, "A Method for the Solution of Certain Non-Linear Problems in Least Squares", Quarterly of Applied Mathematics, Vol.2, No.2, pp.164-168, 1944.

[11] D.Marquardt, "An Algorithm for Least-Squares Estimation of Nonlinear Parameters", SIAM Journal on Applied Mathematics, Vol.11, No.2, pp.431-441, 1963.

[12] S. Chen, S. A. Billings, and P. M. Grant, "Nonlinear system identification using neural networks," Int.I. Conir., Vol.51, No.6, pp.1191-1214, 1990

[13] I. J. Leontaritis and S.A. Billings, "Input-output parametric models for nonlinear systems:PartI: Deterministic nonlinear systems," Ini. J.Contr., Vol.41, No.2, pp.303-328, 1985

[14] L. Ljung, "Syslenz Identijication: Theoryfur the User" Englewood Cliffs,NJ:Prentice-Hall, 1987

[15] H.T. Su and T. J. McAvoy, "Identification of chemical processes using recurrent networks," In: Proc. Amer. Conrr. Conj:, Vol.3, pp.23142319, 1991.

[16] H.T. Su, T. J. McAvoy, and P. Werbo, "Longterm predictions of chemical processes using recurrent neural networks: A parallel training approach," Ind. Eng. Chem. Res., Vol.31, pp.1338-1352, 1992.

[17] K. S. Narendra and K. Parthasarathy, "Identification and control of dynamical systems using neural networks," IEEE Trans. Neural Networks,Vol.1, pp.4-27, 1990.

[18] T. Kdilath, "Linear Systems" Englewood Cliffs, NJ: IPrentice-Hall, 1980.

[19] H. K. Khalil, "Nonlinear Systems" New York: Macmillan, 1992.

[20] A. Toledo, M. Pinzolas, J. J. Ibarrola and G. Lera, "Improvement of the neighborhood based Levenberg-Marquardt algorithm by local adaptation of the learning coefficient," IEEE Transactions on Neural Networks, Vol. 16, No. 4, pp. 988-992, 2005.

[21] J. Rayno, M. F. Iskander and M. H. Kobayashi, "Hybrid Genetic Programming With Accelerating Genetic Algorithm Optimizer for 3-D Metamaterial Design," IEEE Antennas and Wireless Propagation Letters, Vol. 15, pp. 1743-1746, 2016.

[22] Z. Musa, M. Z. Salleh, R. A. Bakar and J. Watada, "GbLN-PSO and Model-Based Particle Filter Approach for Tracking Human Movements in Large View Cases," IEEE T Circ Syst Vid, Vol. 26, No. 8, pp. 1433-1446, 2016. 